\title{
LES ORGANITES ARGYROPHILES SUPERFICIELS DES FORMES LARVAIRES INFESTANTES DE SCHISTOSOMA INTERCALATUM
}

\author{
par Ch. Bayssade-Dufour *, J.-L. Albaret *, H. Picot * et M. Deniau * \\ * Laboratoire de Zoologie (Vers), associé au C.N.R.S., Muséum national d'Histoire naturelle, \\ 43, rue Cuvier, F 75231 Paris Cedex 05. \\ * Laboratoire de Parasitologie, Faculté de Médecine, Broussais-Hôtel Dieu, \\ 15, rue de l'Ecole de Médecine, F 75006 Paris. \\ ** Laboratoire de Parasitologie, Faculté de Médecine de Paris XII, \\ 6, rue du Général-Sarrail, F 94000 Créteil.
}

RESUME. Bien que Schistosoma intercalatum soit actuellement considéré comme une espèce bien individualisée, l'étude détaillée des structures argyrophiles superficielles du miracidium et la chétotaxie de la cercaire ne permettent pas de distinguer ces stades larvaires de ceux de $S$. bovis. Ces caractères mettent donc en évidence l'étroite parenté entre les deux espèces.

Superficial argentophilic structures of miracidium and cercaria of Schistosoma intercalatum.

SUMMARY. Though it is presently admitted that Schistosoma intercalatum is a well individualized species, detailed studies of superficial argentophilic structures of the miracidium and chaetotaxy of the cercaria do not allow these larval stages to be distinguished from those of $\mathrm{S}$. bovis. Therefore this emphasizes the close relationship between the two parasites.

Considéré successivement comme une variété puis comme une sous-espèce de Schistosoma haematobium, un synonyme de $S$. mattheei, un hybride de $S$. haematobium et de $S$. mattheei ou de S. haematobium et de S. bovis, S. intercalatum Fisher, 1934 apparaît actuellement comme une espèce individualisée caractérisée par la taille et la forme des œufs, leur colorabilité $($ Ziehl + ), leur localisation (Mojon et Kien Truong, 1972). Les caractères morphologiques des adultes et des formes larvaires ne permettent guère de distinguer ce parasite d'autres espèces du genre.

Disposant d'une souche isolée à partir d'une malade gabonnaise ayant également séjourné en Côte-d'Ivoire, nous avons pensé qu'il serait intéressant d'étudier les formations argyro-

Accepté le 29 novembre 1979. 
philes superficielles des formes larvaires infestantes, dans le but de les comparer à ceiles d'autres espèces de Schistosomes déjà étudiées à ce point de vue. L'imprégnation argentique des miracidiums a été faite par la méthode de Lynch (1933), celle des cercaires par la technique de Combes, Bayssade-Dufour et Cassone (1976).

\section{Le miracidium.}

Les cellules ciliées au nombre de 22 sont classiquement réparties en quatre étages comprenant d'avant en arrière $6,9,4$ et 3 cellules séparées par trois ceintures: antérieure, moyenne et postérieure (fig. A) :

- le térébratorium porte par hémicorps 9 formations argyrophiles (fig. B);

- la ceinture antérieure comprend 6 papilles ciliées situées dans une échancrure au milieu de la base de chacune des cellules ciliées du $1^{\text {er }}$ étage et 4 formations circulaires par hémicorps dont 2 isolées et 2 contiguës latérales ( $f i g$. A et B) ;

- au niveau de la ceinture moyenne, on observe des papilles ciliées et une petite formation satellite d'une papille ciliée au voisinage de chacun des pores excréteurs. Ceux-ci, au nombre de 2, s'ouvrent latéralement entre les cellules ciliées du $3^{e}$ étage (fig. A).

Le nombre des papilles ciliées de la ceinture moyenne est variable dans une même population. Des variations s'observent également selon l'hôte définitif considéré. Pour des miracidiums provenant d'œufs isolés des selles de la malade, ce nombre varie de 12 à 21 avec une moyenne à $18,14 \pm 0,84$ (pour 21 miracidiums). Après un passage sur Bulinus (Bulinus) forskalii-souris blanche, le nombre des papilles ciliées varie de 17 à 21 avec une moyenne à $19,06 \pm 0,36$ (pour 30 miracidiums).

\section{La cercaire.}

Les miracidiums issus des œufs provenant des selles de la malade sont utilisés pour l'infestation de différents mollusques sains nés au laboratoire:

Bulinus (Bulinus) forskalii (souche originaire du Sénégal), Bulinus (Physopsis) africanus (souche provenant de Tanzanie) et B. (Ph.) globosus (souche originaire du Ghana).

Le pourcentage de mollusques infestés s'est révélé très important (voisin de $100 \%$ ) dans le cas de $B$. (B.) forskalii; par contre, aucun autre mollusque ne s'est montré réceptif au parasite.

\section{- Chétotaxie :}

- Région céphalique : par hémicorps (fig. $C, D, E$ ).

Le cycle $C_{1}$ comporte $1 C_{1} V, 6$ à $8 C_{1} L$ et $1 C_{1} D$. Le cycle $C_{I I}$ se compose d'une $C_{1 I} V$ et $1 \mathrm{C}_{11} \mathrm{D}$. Le cycle $\mathrm{C}_{11}$ est constitué d'une $\mathrm{C}_{11} \mathrm{~V}, 1 \mathrm{C}_{\mathrm{m}} \mathrm{L}$ le plus souvent et $2 \mathrm{C}_{11} \mathrm{D}$.

- Corps: par hémicorps (fig. $C, D, E$ ).

Le cycle $A_{I}$ se compose d'une $A_{1} V$ et d'une $A_{I} D$. Les papilles $A_{I} L$ sont généralement au nombre de $4+1$. Toutefois, ce nombre présente des variations qui n'affectent qu'un seul hémicorps à la fois : $4+2,4+0,3+1,2+1$. Le cycle $\mathrm{A}_{I I}$ comporte, dans la majorité des cas, $1 A_{I I} L$ et $1 A_{I I} D ; 1 A_{I I I} V, 1 A_{H 1} L$ et $2 A_{I I I} D$ forment le cycle $A_{I I I}$. Le cycle $M_{I}$ est constitué le plus souvent par $1 \mathrm{M}_{\mathrm{I}} \mathrm{V}$ (rarement 2 sur un seul hémicorps), $1 \mathrm{M}_{\mathrm{I}} \mathrm{L}$ et $1 \mathrm{M}_{\mathrm{I}} \mathrm{D}$. Le cycle $\mathrm{P}_{\mathrm{II}}$ porte $1 \mathrm{P}_{\mathrm{II}} \mathrm{V}$. Le cycle $\mathrm{P}_{\mathrm{III}}$ se compose d'une $\mathrm{P}_{\mathrm{III}} \mathrm{V}$ et $1 \mathrm{P}_{\mathrm{III}} \mathrm{D}$.

- Acétabulum. On observe, dans la majorité des cas, la disposition classique : $3 \mathrm{~S}_{1}+1 \mathrm{~S}_{11}$ (fig. C). 
- Queue (fig. F, G, H). Le tronc caudal porte, au total, 17 à 25 papilles ventrales et 15 à 21 papilles dorsales. Au niveau du quart postérieur se trouve, ventralement et dorsalement, un groupe de 5 à 7 papilles dont la localisation est constante.

Pour les deux furcas, le nombre des papilles ventrales varie de 4 à 8 et celui des papilles dorsales de 5 à 7.
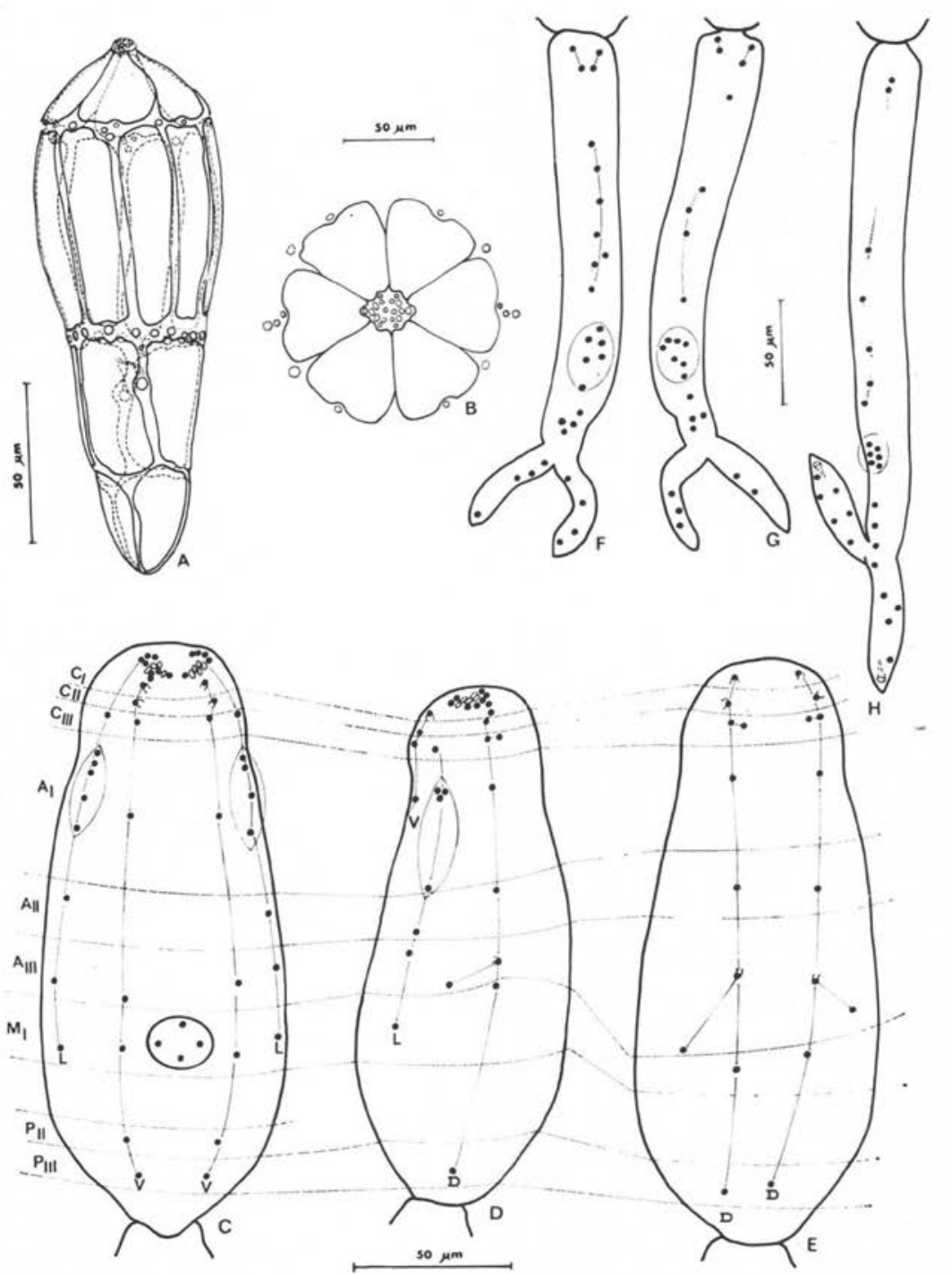

Schistosoma intercalatum. Miracidium: A : vue latérale ; B : vue apicale. Cercaire : corps : C : vue ventrale; D : vue latéro-dorsale; E: vue dorsale; queue: F : vue ventrale; $\mathrm{G}$ : vue dorsale ; $\mathrm{H}$ : vue latéro-dorsale. 


\section{- Miracidium.}

\section{Discussion}

Par le nombre et la disposition des formations argyrophiles de la ceinture antérieure, le miracidium de Schistosoma intercalatum est identique à celui de $S$. haematobium décrit par Capron et coll. (1965) ou à celui de $S$. bovis (Albaret in. litt.) mais se distingue nettement de celui de $S$. mansoni observé par ces mêmes auteurs et par Albaret (1978).

\section{- Cercaire.}

La chétotaxie des cercaires de Schistosoma a été étudiée par Richard (1971) chez Schistosoma mansoni, S. haematobium, S. bovis, S. rodhaini et par Sakamoto et Ishii (1978) chez $S$. japonicum. La comparaison de ces données avec nos observations montre que la chétotaxie de la cercaire de $S$. intercalatum émise par B. (B.) forskalii est identique à celle de $S$. bovis (1). Il resterait à vérifier s'il en est de même avec la cercaire de $S$. intercalatum émise par $B$. $(P h$.) globosus. En effet, l'impossibilité d'obtenir, par hybridation, des miracidiums viables pendant plusieurs générations (Frandsen, 1978) et les faibles différences observées entre certains caractères des souches passant par chacun de ces mollusques (Bjørneboe et Frandsen, 1979) conduisent ces auteurs à penser que $S$. intercalatum représenterait deux espèces morphologiquement identiques mais biologiquement distinctes dont l'existence est liée à l'étroite spécificité mollusque-parasite.

D'après les travaux de Rousset et coll. (1962) et Becquet (1975), S. intercalatum et $S$. bovis sont différenciables par la colorabilité des œufs, mais l'étude des organites argyrophiles superficiels des formes larvaires infestantes apporte un nouvel argument pour souligner l'étroite parenté entre les deux espèces.

\section{Bibliographie}

Albaret J.-L.: Variations du nombre des papilles ciliées chez les miracidiums de différentes souches de Schistosoma mansoni agent de la bilharziose intestinale. C.R. Acad. Sci., 1978, 286, sér. D, 1359-1362.

Becquet R.: Diagnostic parasitologique des bilharzioses intestinales. Ann. Soc. Belg. Méd. Trop., $1975,5,435-447$.

Bjørneboe A., Frandsen F.A.: A comparison of the characteristics of two strains of Schistosoma intercalatum Fisher, 1934 in mice. J. Helminth., 1979, 53, 195-203.

Capron A., Deblock S., Biguet J., Clay A., Adenis L., Vernes A.: Contribution à l'étude expérimentale de la bilharziose à Schistosoma haematobium. Bull. O.M.S., 1965, 32, 755-778.

Combes C., Bayssade-Dufour Ch., Cassone J.: Sur l'imprégnation et le montage des cercaires pour l'étude chétotaxique. Ann. Parasit. Hum. Comp., 1976, 51, 399-400.

Frandsen F.A.: Hybridization between different strains of Schistosoma intercalatum Fisher, 1934 from Cameroun and Zaïre. J. Helminth., 1978, 52, 11-22.

Lynch J.E.: The miracidium of Heronimus chelydrae, Mac Callum. Quart. J. Microsc. Sci., 1933, 76, 13-33.

Mojon M., Kien-Truong T.: Evolution du concept Schistosoma intercalatum. Lyon Médical, 1972, 228, 331-337.

Richard J.: La chétotaxie des cercaires. Valeur systématique et phylétique. Mém. Mus. nat. Hist. nat., nelle Ser., sér. A, Zool., 1971, 67, 179 p.

Rousset J.-J., Houin R., Buttner A.: Acido-alcoolo-resistance de divers œufs de Schistosomes. Modification de la technique de Brygoo, Capron et Randriamala. Ann. Parasit. Hum. Comp., 1962, 37, 866-869.

Sakamoto K., Ishii Y.: Scanning electron microscope observations on miracidium, cercaria and cercarial papillar patterns of Schistosoma japonicum. J. Parasit., 1978, 64, 59-68.

(1) Souche originaire de Sardaigne. Cercaires émises par Bulinus truncatus. 